\title{
BACTERIAL CONTAMINATION OF NYLON CORNEAL SUTURES
}

\author{
C. J. HEAVEN ${ }^{1}$, C. R. N. DAVISON ${ }^{2}$ and P. M. COCKCROFT ${ }^{3}$ \\ Manchester, Southampton and Portsmouth
}

\begin{abstract}
SUMMARY
We report the findings of a prospective study into the bacterial contamination of monofilament nylon sutures removed from corneal wounds following cataract surgery. Sutures were classified as tight, loose or broken at the time of removal. Loose and broken sutures showed significantly more bacterial contamination than tight sutures $(p<0.001$, chi squared). Positive cultures were obtained from $2(6.2 \%)$ of 32 tight sutures, $14(38.9 \%)$ of 36 loose sutures and $11(37.9 \%)$ of 29 broken sutures. Staphylococcus epidermidis was the most commonly isolated organism (isolated in pure growth from $22(81.5 \%)$ of 27 positive cultures). These findings may explain the occasional association of biodegraded corneal monofilament nylon sutures and suppurative keratitis and highlight the potential risk of seeding a suture track infection at the time of suture removal. They also emphasise the need for prophylactic topical antibiotic when removing biodegraded sutures.
\end{abstract}

Conventional extracapsular cataract surgery is frequently performed via a corneal incision. Monofilament nylon (MFN) is a popular suture material for closing such wounds. However, redundant MFN sutures may occasionally facilitate the development of suppurative keratitis.' The prevalence of bacterial contamination of retained MFN sutures, and hence the rissk of corneal infection, is unknown. We have therefore conducted a prospective study of the bacterial contamination of MFN sutures at the time of their removal from the corneal wounds of cataract surgery.

\footnotetext{
From: 'Manchester Royal Eye Hospital. Manchester: ${ }^{2}$ Department of Ophthalmology, Southampton General Hospital, Southampton; 'Public Health Laboratory, St Mary's General Hospital, Portsmouth, UK. Study conducted at Department of Ophthalmology, Queen Alexandra Hospital, Portsmouth PO6 3LY, UK.

Correspondence to: Mr C. J. Heaven, Manchester Royal Eye Hospital, Oxford Road, Manchester M13 9WH, UK.
}

\section{PATIENTS AND METHODS}

Individual interrupted $10 / 0$ monofilament nylon sutures were harvested from the corneal wounds of eyes that had undergone extracapsular cataract extraction. Sutures were classified as tight, loose or broken. Thirty-two tight, 36 loose sutures and 29 broken sutures were collected. The approximate size of these groups was determined prior to the start of the study following discussions with a microbiologist and a statistician. Suture removal was based on clinical need. For tight sutures removal was for relief of surgically induced astigmatism and was usually performed 2-3 months post-operatively. For loose and broken sutures removal was for relief of irritation and corneo-conjunctival inflammation. Sutures were removed at the slit lamp using a sterile technique. Tight and loose sutures were cut with a 25 gauge needle, mounted on a $2 \mathrm{ml}$ syringe as a handle. Sutures were extracted from the cornea using flamesterilised microforceps and immediately placed into Brain Heart Infusion broth (Unipath Ltd) which was incubated at $37^{\circ} \mathrm{C}$ for 24 hours. Each broth was then subcultured onto blood agar and chocolate agar incubated at $37^{\circ} \mathrm{C}$ in $\mathrm{CO}_{2}$, blood agar incubated at $37^{\circ} \mathrm{C}$ anaerobically and glucose Sabaraud agar in air at room temperature. All plates were incubated for 5 days and examined daily for bacterial and fungal growth. Isolates were identified by standard techniques; sensitivity tests were performed using comparative disc diffusion ('Stokes') method.

To avoid contact between the arms of the microforceps and the inside of the culture bottle, and to overcome the reluctance of sutures to transfer from forceps to broth, sutures were placed onto the rubber lining of the culture bottle cap. Having tightly replaced this cap the suture was shaken into the broth itself. Only one suture per patient was included in the study. Patients with suspected corneal infection 
Table I. Bacterial contamination of corneal monofilament nylon sutures after cataract surgery, and organisms involved

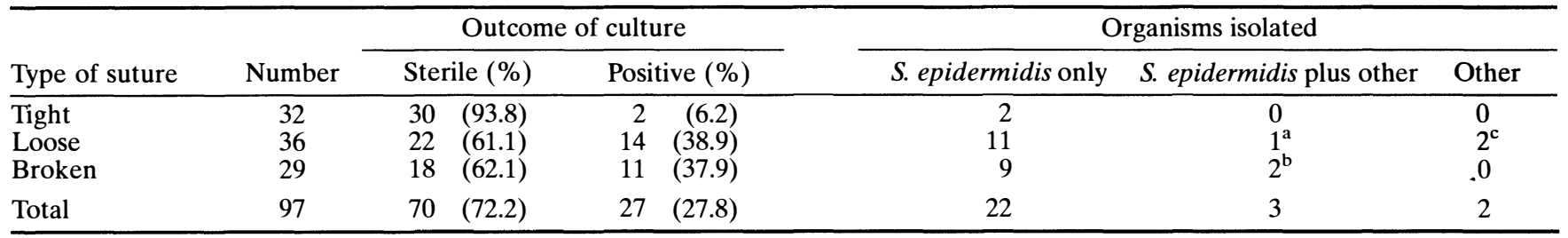

${ }^{\mathrm{a}} S$. epidermidis (three types) and Bacillus sp.

${ }^{\mathrm{b}}$ One suture grew $S$. epidermidis and Group B Streptococcus, one grew S. epidermidis (two types) and Streptococcus mitis.

${ }^{\mathrm{c} O n e}$ suture grew a viridans-type streptococcus and one grew a Gram-negative bacillus.

or use of antibiotic within the last 2 weeks were excluded. Conjunctival swabs were not taken.

\section{RESULTS}

A positive bacterial culture was obtained from 2 $(6.2 \%)$ of the 32 tight sutures, from $14(38.9 \%)$ of the 36 loose sutures and from $11(37.9 \%)$ of the 29 broken sutures (Table I). The incidences of bacterial contamination of loose and of broken sutures were both significantly greater than that of tight sutures $(p<0.001$ in each case, chi-squared). The contamination rates of loose and broken sutures were not significantly different from each other $(p>0.5)$.

The most frequently identified organism was Staphylococcus epidermidis. This was isolated in 25 $(92.6 \%)$ of the 27 culture-positive cases. In 22 of these cases this was the only organism isolated, although in 1 case from each group two different strains of $S$. epidermidis were isolated from a single suture. In 5 cases other organisms were isolated. One loose suture yielded a mixed growth of three strains of $S$. epidermidis with Bacillus sp. A viridans-type streptococcus and a Gram-negative bacillus (which failed to grow for full identification) were isolated from one loose suture each. One broken suture yielded both $S$. epidermidis and a Group B betahaemolytic streptococcus and one a mixture of two types of S. epidermidis and Streptococcus mitis. All organisms were sensitive to at least one of the following antibiotics: chloramphenicol, gentamicin, erythromycin and flucloxacillin. No patient from whom sutures had been collected suffered subsequent suppurative keratitis.

\section{DISCUSSION}

Monofilament nylon is used widely in the United Kingdom for the closure of corneal incisions after cataract surgery. It offers high elasticity, sustained tensile strength and little tissue reaction within the cornea. Although classified as a non-absorbable material it does slowly biodegrade. ${ }^{1,2}$ Amide linkages within the polymer udergo hydrolysis mediated by lysozymal enzymes. ${ }^{1,2}$ This results in fretting and cracking of sutures with progressive loss of tensile strength, and in time sutures loosen and may break. $^{1,3}$ Occasionally such biodegraded MFN sutures provide a nidus for the development of suppurative keratitis. ${ }^{1}$ This complication has also been reported shortly after suture removal, presumably due to seeding of microorganisms into the suture track. ${ }^{4}$ These phenomena suggest that retained biodegraded corneal MFN sutures may harbour bacteria. Our findings confirm this suspicion and quantify the problem.

Most isolates were of low-pathogenicity bacteria which commonly colonise the skin and conjunctival sac. Their significance is difficult to evaluate but the ability of such organisms to cause infection when associated with foreign material is well recognised and especially the affinity of $S$. epidermidis for synthetic material such as plastics. ${ }^{5,6,8-11}$ In addition, $S$. epidermidis contamination of synthetic material has been shown to facilitate colonisation by other organisms including Pseudomonas aeruginosa. ${ }^{7-10}$ Gram-negative bacilli such as Pseudomonas spp. (and Gram-positive streptococci) are among the most virulent corneal pathogens and are capable of producing severe suppurative keratitis. The increased incidence of bacterial contamination of loose and broken sutures $(38.9 \%$ and $37.9 \%$ respectively) over that of tight sutures $(6.2 \%)$ therefore suggests that the former are at increased risk of infective complications.

Broken sutures tend to be removed late, for relief of ocular irritation, and are usually biodegraded. Broken sutures are also often associated with a local corneal epithelial defect. This in combination with suture contamination provides conditions particularly favourable for the development of suppurative keratitis. Loose sutures also demonstrated frequent contamination which may relate both to biodegradation and to their tendency to accumulate mucus. Tight corneal sutures are usually free of bacterial contamination. This is probably because they are removed relatively soon after surgery for relief of induced astigmatism. At this stage they are unlikely to have undergone significant biodegradation and are covered by corneal epithelium. The latter feature is confirmed clinically by absence of staining on instillation of fluorescein.

These findings provide a further indication that corneal MFN sutures should be removed once 
wound healing is complete and before significant suture biodegradation occurs. However, the optimal regime of prophylactic topical antibiotic at the time of suture removal remains uncertain. In the light of our findings we feel that a single dose of topical antibiotic ointment may suffice when removing tight sutures. The aim here is to prevent infection whilst the small iatrogenic corneal abrasion heals. A short course of topical antibiotic may, however, be advisable after removing loose or broken sutures to guard against the possibility of having implanted suture-borne bacteria into the cornea at the time of removal. Sutureless cataract surgery will, of course, dispense with the problem of suture contamination altogether.

This study was instigated following an idea by $\mathrm{Mr} \mathrm{D}$. L. Boase, Consultant Ophthalmic Surgeon, Queen Alexandra Hospital, Portsmouth.

Key words: Bacterial infections, Cornea, Nylon, Sutures.

\section{REFERENCES}

1. Acheson JF, Lyons CJ. Ocular morbidity due to monofilament nylon corneal sutures. Eye 1991;5: 106-12.

2. Hayasaka S, Ishiguro S, Shiono T, Okabe H, Mizuno K. Scanning electron microscopic study of nylon degradation by ocular tissue extracts. Am J Ophthalmol 1982;93:111-7.
3. Jackson H, Bosanquet R. Should nylon corneal sutures be routinely removed? $\mathrm{Br} \mathrm{J}$ Ophthalmol 1991;75: 663-4.

4. Heaven CJ, Boase DL. Suppurative keratitis with endophthalmitis due to biodegraded full thickness monofilament nylon corneal sutures. Eur J Implant Refract Surg 1993;5:164-8.

5. Raskin EM, Speaker MG, McCormick SA, Wong D, Menikoff JA, Pelton-Henrion K. Influence of haptic materials on the adherence of staphylococci to intraocular lenses. Arch Ophthalmol 1993;111:250-3.

6. Portoles M, Retojo MF, Leong FL. Reduced bacterial adhesion to heparin-surface-modified intraocular lenses. J Cataract Refract Surg (US) 1993;19:755-9.

7. Chang CC, Merritt K. Effect of Staphylococcus epidermidis on adherence of Pseudomonas aeruginosa and Proteus mirabilis to polymethyl methacrylate (PMMA) and gentamicin-containing PMMA. J Orthop Res 1991;9:284-8.

8. Tsai CL, Liu TK, Hung MH. Glycocalyx production and adherence of Staphylococcus to biomaterials. Acta Med Okayama 1992;464:11-6.

9. Muller E, Takeda S, Shiro H, Goldmann, D, Pier GB. Occurrence of capsular polysaccharide/adhesin among clinical isolates of coagulase-negative staphylococci. J Infect Dis 1993;168:1211-8.

10. Chang CC, Merritt K. Microbial adherence on poly(methyl methacrylate) (PMMA) surfaces. J Biomed Mater Res 1992;26:197-207.

11. Bayston R, Rodgers J. Production of extra-cellular slime by Staphylococcus epidermidis during stationary phase of growth: its association with adherence to implantable devices. J Clin Pathol 1990;43:866-70. 